\title{
Bounds for the Solutions of Second-Order Linear Difference Equations
}

\author{
F. W. J. Olver \\ Institute for Basic Standards, National Bureau of Standards, Washington, D.C. 20234
}

(August 22, 1967)

\begin{abstract}
Simple bounds are established for the solutions of second-order homogeneous linear difference equations in ranges in which the solutions are exponential in character. The results are applied to a recent algorithm for the computation of subdominant solutions of second-order linear difference equations, homogeneous or otherwise. Strict and extremely realistic bounds are obtained for the truncation error associated with the algorithm in a number of examples, including Anger-Weber functions, Struve functions, and the solution of a differential equation in Chebyshev series.
\end{abstract}

Key Words: Chebyshev series, difference equations, error bounds, Miller algorithm, recurrence relations, special functions.

\section{Introduction}

Consider the difference equation

$$
p_{r+1}-b p_{r}+p_{r-1}=0,
$$

in which $b$ is a real constant exceeding 2. The solution may be expressed as

$$
p_{r}=\frac{p_{1}-\lambda_{2} p_{0}}{\lambda_{1}-\lambda_{2}} \lambda_{1}^{r}+\frac{\lambda_{1} p_{0}-p_{1}}{\lambda_{1}-\lambda_{2}} \lambda_{2}^{r}
$$

where $p_{0}$ and $p_{1}$ are at our disposal, and

$$
\lambda_{1}=\frac{1}{2} b+\sqrt{\frac{1}{4} b^{2}-1}, \quad \lambda_{2}=\frac{1}{2} b-\sqrt{\frac{1}{4} b^{2}-1} .
$$

Eventually $\left|p_{r}\right|$ grows in proportion to $\lambda_{1}^{r}$ as $r \rightarrow \infty$, unless $p_{1}=\lambda_{2} p_{0}$. The larger the value of $b$, the faster is the rate of growth.

Now consider the equation

$$
p_{r+1}-b_{r} p_{r}+p_{r-1}=0,
$$

in which $b_{1}, b_{2}, b_{3}, \ldots$ is a given sequence of real numbers, not necessarily monotonic, each of which exceeds the $b$ of eq (1.01). If the same values of $p_{0}$ and $p_{1}$ are used for the two difference equations, then it is reasonable to expect-and not difficult to provethat the solution of (1.02) generally increases at a

\footnotetext{
${ }^{1}$ Figures in brackets indicate the literature references at the end of this paper.
}

faster rate than that of (1.01). Similarly, if, for every $r, b_{r}<B$, where $B$ is another constant, then we expect the solution of (1.02) to grow at a slower rate than the solution of

$$
p_{r+1}-B p_{r}+p_{r-1}=0
$$

having the same initial values.

The first purpose of the present paper is to generalize these intuitive results and present them as precise theorems. We shall allow the coefficient of $p_{r-1}$ in (1.02) to take values other than unity, and we shall not insist that the solutions of difference equations under comparison agree at both $r=0$ and $r=1$. Some related results for the tails of continued fractions have been given by Blanch [1] ${ }^{1}$ and Merkes [2].

The second purpose of this paper is to apply these theorems to a recent algorithm for computing subdominant solutions of homogeneous or inhomogeneous second-order difference equations [3], with a view to obtaining bounds for the truncation error.

\section{Comparison Theorem}

THEOREM 1. Let

$$
\begin{array}{r}
\mathrm{q}_{\mathrm{r}+1}=\beta_{\mathrm{r}} \mathrm{q}_{\mathrm{r}}-\alpha_{\mathrm{r}} \mathrm{q}_{\mathrm{r}-1}, \\
\mathrm{Q}_{\mathrm{r}+1}=\mathrm{B}_{\mathrm{r}} \mathrm{Q}_{\mathrm{r}}-\mathrm{A}_{\mathrm{r}} \mathrm{Q}_{\mathrm{r}-1},
\end{array}
$$

where $\left\{\alpha_{\mathrm{r}}\right\},\left\{\beta_{\mathrm{r}}\right\},\left\{\mathrm{A}_{\mathrm{r}}\right\},\left\{\mathrm{B}_{\mathrm{r}}\right\}, \mathrm{r}=1,2, \ldots$, are sequences of numbers such that 


$$
\mathrm{A}_{\mathrm{r}} \geqq \alpha_{\mathrm{r}} \geqq 0, \quad \mathrm{~B}_{\mathrm{r}}-\mathrm{A}_{\mathrm{r}} \geqq \beta_{\mathrm{r}}-\alpha_{\mathrm{r}} \geqq 1 .
$$

If

$\mathrm{Q}_{1}-\mathrm{q}_{1} \geqq \mathrm{Q}_{0}-\mathrm{q}_{0} \geqq 0$, and $\mathrm{q}_{1} \geqq \max \left(\mathrm{q}_{0}, 0\right)$,

then $\mathrm{q}_{\mathrm{r}}$ and $\mathrm{Q}_{\mathrm{r}}$ are nondecreasing functions of $\mathrm{r}$, and $\mathrm{q}_{\mathrm{r}} \leqq \mathrm{Q}_{\mathrm{r}}, \mathrm{r}=0,1, \ldots$.

To establish this result, we begin with the identity

$$
q_{r+1}-q_{r}=\left(\beta_{r}-\alpha_{r}-1\right) q_{r}+\alpha_{r}\left(q_{r}-q_{r-1}\right),
$$

and observe that both $\beta_{r}-\alpha_{r}-1$ and $\alpha_{r}$ are nonnegative. From (2.04) we see that $q_{1} \geqq 0$ and $q_{1}-q_{0} \geqq 0$. Hence from (2.05), with $r=1$, it follows that $q_{2}-q_{1} \geqq 0$. Continuing this argument by induction, we see that $q_{r+1} \geqq q_{r}, r=0,1, \ldots$. Similarly from the identity

$$
Q_{r+1}-Q_{r}=\left(B_{r}-A_{r}-1\right) Q_{r}+A_{r}\left(Q_{r}-Q_{r-1}\right), \quad \text { (2.06) }
$$

we deduce that $\left\{Q_{r}\right\}$ is a nondecreasing sequence.

Next, subtraction of (2.05) and (2.06) yields

$$
\begin{aligned}
\left(Q_{r+1}-q_{r+1}\right)-\left(Q_{r}-q_{r}\right) & =\left(\beta_{r}-\alpha_{r}-1\right)\left(Q_{r}-q_{r}\right) \\
& +\left\{\left(B_{r}-A_{r}\right)-\left(\beta_{r}-\alpha_{r}\right)\right\} Q_{r} \\
& +\alpha_{r}\left\{\left(Q_{r}-q_{r}\right)-\left(Q_{r-1}-q_{r-1}\right)\right\} \\
& +\left(A_{r}-\alpha_{r}\right)\left(Q_{r}-Q_{r-1}\right) .
\end{aligned}
$$

Assume that $r \geqq 1$ and $Q_{r}-q_{r} \geqq Q_{r-1}-q_{r-1} \geqq 0-$ as is certainly the case when $r=1$ in consequence of (2.04). Then using (2.03) and the facts that $Q_{r} \geqq 0$ and $Q_{r}-Q_{r-1} \geqq 0$, we deduce that $\left(Q_{r+1}-q_{r+1}\right)-\left(Q_{r}-q_{r}\right)$ $\geqq 0$. Hence by induction $Q_{r} \geqq q_{r}$. This completes the proof.

\section{Bounds for the Solutions When the $a_{r}$ Are Nonnegative}

THEOREM 2. Let

$$
\mathrm{p}_{\mathrm{r}+1}=\mathrm{b}_{\mathrm{r}} \mathrm{p}_{\mathrm{r}}-\mathrm{a}_{\mathrm{r}} \mathrm{p}_{\mathrm{r}-1}
$$

where $\left\{a_{\mathrm{r}}\right\}$ and $\left\{\mathrm{b}_{\mathrm{r}}\right\}, \mathrm{r}=1,2, \ldots$, are sequences of numbers such that

$$
\mathrm{b}_{\mathrm{r}} \geqq 1+\mathrm{a}_{\mathrm{r}} \geqq 1,
$$

and suppose that

$$
\mathrm{p}_{0}>0, \quad \kappa \equiv \mathrm{p}_{1} / \mathrm{p}_{0} \geqq 1 .
$$

Then $\mathrm{p}_{\mathrm{r}}$ is a nondecreasing function of $\mathrm{r}$, and

$$
\begin{array}{r}
\mathrm{p}_{0}\{\min (\kappa, \lambda)\}^{\mathrm{r}} \leqq \mathrm{p}_{\mathrm{r}} \leqq \mathrm{p}_{0}\{\max (\kappa, \Lambda)\}^{\mathrm{r}} \\
(\mathrm{r}=0,1, \ldots) .
\end{array}
$$

Here $\lambda, \Lambda$ are the largest roots of the equations

$$
\lambda^{2}-\mathrm{b} \lambda+\mathrm{a}=0, \quad \Lambda^{2}-\mathrm{B} \Lambda+\mathrm{A}=0,
$$

respectively, and

$$
\begin{array}{ll}
a=\inf _{r \geqq 1} a_{r}, & b=a+\inf _{r \geqq 1}\left(b_{r}-a_{r}\right), \\
A=\sup _{r \geqq 1} a_{r}, & B=A+\sup _{r \geqq 1}\left(b_{r}-a_{r}\right) .
\end{array}
$$

Before entering into the proof of this theorem, we observe that the numbers $b$ and $a$ always exist and are subject to the inequalities

$$
b \geqq 1+a \geqq 1 .
$$

Moreover,

$\lambda=\frac{1}{2} b+\sqrt{\frac{1}{4} b^{2}-a} \geqq \frac{1}{2} a+\frac{1}{2}+\left|\frac{1}{2} a-\frac{1}{2}\right| \geqq \max (a, 1)$.

On the other hand, either or both of the sequences $\left\{a_{r}\right\}$ and $\left\{b_{r}-a_{r}\right\}$ may be unbounded, causing either $B$ or both $B$ and $A$ to be infinite: in this event $\Lambda$ is undefined and the second of the inequalities (3.04) is inapplicable. Except in these cases, we have ${ }^{2}$

$$
A \geqq a, \quad B \geqq b, \quad \Lambda \geqq A, \quad \Lambda \geqq \lambda .
$$

That $p_{r}$ is a nondecreasing function of $r$ is an immediate consequence of (3.02), (3.03), and Theorem 1 . We shall establish the inequalities (3.04) in turn by further use of this theorem.

Suppose first that $\kappa \geqq \lambda$. Define $q_{r}=p_{0} \lambda^{r}$. Then $q_{0}=p_{0}, q_{1}=p_{0} \lambda$ and, in consequence of (3.05),

$$
q_{r+1}=b q_{r}-a q_{r-1} \quad(r \geqq 1) .
$$

We apply Theorem 1, with (3.11) filling the role of (2.01), and (3.01) that of (2.02). Conditions (2.03) are satisfied in consequence of the definitions of $a$ and $b$, and (2.04) is satisfied since $\kappa \geqq \lambda \geqq 1$. Therefore $p_{r} \geqq q_{r}=p_{0} \lambda^{r}$, in agreement with the first of (3.04).

Now consider the case when $\kappa<\lambda$. Define $q_{r}=p_{0} \kappa^{r}$. Then $q_{0}=p_{0}, q_{1}=p_{1}$, and

$$
q_{r+1}=\tilde{b} q_{r}-\tilde{a} q_{r-1} \quad(r \geqq 1),
$$

where $\tilde{a}=\min (a, \kappa)$ and $\tilde{b}=\kappa+\tilde{a} \kappa^{-1}$. The desired result $p_{r} \geqq q_{r}$ will follow if Theorem 1 can be applied to eqs (3.12) and (3.01). Conditions (2.04) are clearly satisfied; the remaining conditions are

$$
a_{r} \geqq \tilde{a} \geqq 0, \quad b_{r}-a_{r} \geqq \tilde{b}-\tilde{a} \geqq 1 .
$$

The first two inequalities follow from the definitions of $a$ and $\tilde{a}$. And since

$$
\tilde{b}-\tilde{a}-1=(\kappa-1)\left(1-\tilde{a} \kappa^{-1}\right) \geqq 0, \quad b-a \leqq b_{r}-a_{r},
$$

\footnotetext{
${ }^{2}$ The last of (3.10) may be verified by expressing $2 \lambda=b+\left(b^{2}-4 b+4 c\right)^{1 / 2}$, where
} $c \equiv \inf \left(b_{r}-a_{r}\right) \geqq 1$, showing that $\partial \lambda / \partial b$ and $\partial \lambda / \partial c$ are both positive. 
it will suffice to show that

$$
b-a \geqq \tilde{b}-\tilde{a} .
$$

If $\tilde{a}=\kappa$, then $\tilde{b}=\kappa+1$ and (3.13) follows immediately from (3.08). If, on the other hand, $\tilde{a}=a<\kappa$, then

$$
\begin{aligned}
(b-a)-(\tilde{b}-\tilde{a}) & =b-\kappa-a \kappa^{-1} \\
& =\lambda+\frac{a}{\lambda}-\left(\kappa+\frac{a}{\kappa}\right) \\
& =(\lambda-\kappa)\left(1-\frac{a}{\lambda \kappa}\right)>0,
\end{aligned}
$$

since $a<\kappa$ and $1 \leqq \kappa<\lambda$. This completes the proof of the left-hand inequality in (3.04).

To establish the right-hand inequality in (3.04), suppose first that $\kappa \leqq \Lambda$. Defining $Q_{r}=p_{0} \Lambda^{r}$, we have $Q_{0}=p_{0}, Q_{1}=p_{0} \Lambda$, and

$$
Q_{r+1}=B Q_{r}-A Q_{r-1} \quad(r \geqq 1) .
$$

It is readily verified that Theorem 1 is applicable to eqs (3.01) and (3.14). Hence $p_{r} \leqq p_{0} \Lambda^{r}$, in agreement with (3.04).

Now consider the case when $\kappa>\Lambda$. Define $Q_{r}=p_{0} \kappa^{r}$, so that $Q_{0}=p_{0}, Q_{1}=p_{1}$, and

$$
Q_{r+1}=\tilde{B} Q_{r}-A Q_{r-1} \quad(r \geqq 1),
$$

where $\tilde{B}=\kappa+A \kappa^{-1}$. The desired result $p_{r} \leqq Q_{r}$ will follow immediately if Theorem 1 is applicable to (3.01) and (3.15). Conditions (2.04) are obviously satisfied. Conditions (2.03) demand that

$$
A \geqq a_{r} \geqq 0, \quad \tilde{B}-A \geqq b_{r}-a_{r} \geqq 1 .
$$

In consequence of (3.02) and (3.07), these inequalities will follow if we can show that $\vec{B} \geqq B$. Now

$$
\tilde{B}-B=\kappa+\frac{A}{\kappa}-\left(\Lambda+\frac{A}{\Lambda}\right)=(\kappa-\Lambda)\left(1-\frac{A}{\Lambda \kappa}\right) .
$$

This is nonnegative because $\kappa>\Lambda, \kappa \geqq 1$, and $\Lambda \geqq A$; compare (3.10). The proof of Theorem 2 is now complete.

\section{Bounds for the Solutions When the $a_{r}$ Are Nonpositive}

If the quantities $a_{r}$ are negative then Theorem 2 is inapplicable. The analysis of this case is somewhat easier, however, and Theorem 1 is not needed. For convenience, we replace $a_{r}$ by $-a_{r}$.
THEOREM 3. Let

$$
p_{r+1}=b_{r} p_{r}+a_{r} p_{r-1}
$$

where

$\mathrm{a}_{\mathrm{r}} \geqq 0, \quad \mathrm{~b}_{\mathrm{r}} \geqq 0, \quad \mathrm{p}_{0}>0, \quad \kappa \equiv \mathrm{p}_{1} / \mathrm{p}_{0} \geqq 1$.

Then

$$
\begin{array}{r}
\mathrm{p}_{0}\{\min (\kappa, \lambda)\}^{\mathrm{r}} \leqq \mathrm{p}_{\mathrm{r}} \leqq \mathrm{p}_{0}\{\max (\kappa, \Lambda)\}^{\mathrm{r}} \\
(\mathrm{r}=0,1, \ldots),
\end{array}
$$

where $\lambda, \Lambda$ are the largest roots of the equations

$$
\lambda^{2}-\mathrm{b} \lambda-\mathrm{a}=0, \quad \Lambda^{2}-\mathrm{B} \Lambda-\mathrm{A}=0,
$$

respectively, and

$$
\begin{gathered}
a=\inf _{r \geqq 1} a_{r}, \quad b=\inf _{r \geqq 1} b_{r}, \\
A=\sup _{r \geqq 1} a_{r}, \quad B=\sup _{r \geqq 1} b_{r} .
\end{gathered}
$$

To prove the left-hand inequality in (4.03) when $\kappa \geqq \lambda$, assume that $p_{r} \geqq p_{0} \lambda^{r}$ and $p_{r-1} \geqq p_{0} \lambda^{r-1}$, - as is the case when $r=1$. Then from (4.01) we have

$$
p_{r+1} \geqq b p_{0} \lambda^{r}+a p_{0} \lambda^{r-1}=p_{0} \lambda^{r+1},
$$

as required.

Now consider the case when $\kappa<\lambda$. The roots of the quadratic equation $x^{2}-b x-a=0$ are $\lambda$ and $-a / \lambda$. Since $-a / \lambda<\kappa<\lambda$, it follows that

$$
\kappa^{2}-b \kappa-a<0 .
$$

Assume that $p_{r} \geqq p_{0} \kappa^{r}$ and $p_{r-1} \geqq p_{0} \kappa^{r-1}-$ as is the case when $r=1$. Then from (4.01), (4.05), and (4.07), we derive

$$
p_{r+1} \geqq b p_{0} \kappa^{r}+a p_{0} \kappa^{r-1}>p_{0} \kappa^{r+1} .
$$

The right-hand inequality in (4.03) may be established in a similar way.

\section{Anger-Weber Functions}

In the remaining part of this paper we show how the preceding theorems may be applied to the truncation errors associated with the algorithm of [3].

Consider first the computation of the Anger-Weber function $\mathbf{E}_{r}(1)$ given in [3], section 6, Example 1. The truncation error of the approximation $y_{r}^{(14)}$ is given by ${ }^{3}$

${ }^{3}[3]$, section 5 . 


$$
\epsilon_{r}^{(14)} \equiv \mathbf{E}_{r}(1)-y_{r}^{(14)}=E_{14} p_{r}
$$

where

$$
E_{14}=\sum_{s=14}^{\infty} \frac{e_{s}}{p_{s} p_{s+1}}
$$

$p_{r}$ satisfies (3.01) above with $a_{r}=1, b_{r}=2 r, p_{0}=0$, $p_{1}=1$, and

$$
e_{r}=e_{r-1}-d_{r} p_{r}, \quad d_{r}=-(2 / \pi)\left\{1-(-1)^{r}\right\},
$$

with $e_{0}=\mathbf{E}_{0}(1)$.

We apply Theorem 2 of section 3 to the sequence $\left\{p_{r+14}\right\}$, using the numerical entries given in [3], table 1. Working to five significant figures (for the sake of illustration), we have

$$
\begin{gathered}
\kappa=p_{15} / p_{14}=1.1127 \times 10^{15} /\left(3.9793 \times 10^{13}\right)=27.962, \\
a=1, \quad b=1+\inf _{r \geqq 15}(2 r-1)=30 .
\end{gathered}
$$

Hence

$$
\lambda^{2}-30 \lambda+1=0, \quad \lambda=29.967>\kappa .
$$

Therefore

$$
p_{r} \geqq p_{14} \kappa^{r-14} \quad(r \geqq 14) .
$$

Next, from (5.03) we have

$$
e_{s}=e_{14}+(4 / \pi)\left(p_{15}+p_{17}+\ldots+p_{s^{\prime}}\right) \quad(s>14),
$$

where $s^{\prime}=s$ if $s$ is odd, and $s^{\prime}=s-1$ if $s$ is even. Since $p_{s}$ is a nondecreasing function of $s$ (Theorem 2), we have

$$
e_{s} \leqq e_{14}+(4 / \pi)(s-14) p_{s} \quad(s \geqq 14) .
$$

Application of this inequality to (5.02) gives

$$
E_{14} \leqq e_{14} \sum_{s=14}^{\infty} \frac{1}{p_{s} p_{s+1}}+\frac{4}{\pi}\left(\frac{1}{p_{16}}+\frac{2}{p_{17}}+\frac{3}{p_{18}}+\ldots\right) .
$$

Substituting (5.04) on the right-hand side of (5.07), summing the resulting series and using numerical entries given in [3], table 1, we obtain

$$
E_{14} \leqq \frac{e_{14}}{p_{14}^{2}\left(\kappa-\kappa^{-1}\right)}+\frac{4}{\pi p_{14}(\kappa-1)^{2}}=8.824 \times 10^{-17}
$$

The actual value of $E_{14}$ was estimated in [3] to be
$8.248 \times 10^{-17}$ by direct numerical summation of the terms in the expansion (5.02). Our strict bound (5.08) exceeds this estimate by about 7 percent, which is very satisfactory in view of the somewhat crude step leading from (5.05) to (5.06).

Strict bounds for the errors $\epsilon_{r}^{(14)}(r \leqq 14)$ may be computed by substituting the numerical value (5.08) for $E_{14}$ in (5.01) and using the values of $p_{r}$ given in [3], table 1. Obviously they, too, will overestimate the actual truncation errors by about 7 percent.

\section{Struve Functions}

As a second example, consider the Struve function $\mathbf{H}_{r}(0.1)$ computed in [3], section 6, Example 2. The relevant formulas in this case are

$$
\epsilon_{r}^{(15)}=\mathbf{H}_{r}(0.1)-y_{r}^{(15)}=E_{15} p_{r}, \quad E_{15}=\sum_{s=15}^{\infty} \frac{e_{s}}{p_{s} p_{s+1}},
$$

and

$$
p_{r+1}=20 r p_{r}-p_{r-1}, \quad e_{r}=e_{r-1}-\frac{(0.05)^{r}}{\sqrt{\pi} \Gamma\left(r+\frac{3}{2}\right)} p_{r}
$$

Applying Theorem 2 to the sequence $\left\{p_{r+15}\right\}$ using the numerical entries in [3], table 2, we have

$$
\begin{aligned}
& \kappa=p_{16} / p_{15}=299.99643 \ldots ., \\
& a=1, \quad b=1+\inf _{r \geqq 16}(20 r-1)=320, \\
& \lambda=160+\sqrt{25599}>\kappa .
\end{aligned}
$$

Hence

$$
p_{r} \geqq p_{15} \kappa^{r-15} \quad(r \geqq 15) .
$$

Inspection of table 2 of [3] suggests that

$$
\left|e_{r}\right| \leqq e_{15} \quad(r \geqq 15) .
$$

Deferring the proof of this inequality for the moment and substituting (6.03) and (6.04) in the second of (6.01), we derive

$$
\left|E_{15}\right| \leqq \frac{e_{15}}{p_{15}^{2}\left(\kappa-\kappa^{-1}\right)}=(0.23277966 \ldots) \times 10^{-62}
$$

The actual value of $E_{15}$ may be estimated by computation of $p_{17}, p_{18}, \ldots$ and direct summation of the expansion in (6.01). This gives $(0.23277$ 943 . . .) $\times 10^{-62}$. Therefore strict bounds for $\epsilon_{r}^{(15)}(r \leqq 15)$ computed from (6.05) and the first of (6.01) will exceed 
the estimated errors by less than one part in $10^{6}$, a striking example of the power of Theorem 2.

It remains to establish (6.04). Write

$$
u_{r}=(0.05)^{r} p_{r} /\left\{\sqrt{\pi} \Gamma\left(r+\frac{3}{2}\right)\right\},
$$

so that

$$
e_{r}=e_{15}-u_{16}-u_{17}-\ldots .-u_{r} \quad(r \geqq 16) .
$$

The $p_{r}$ and $u_{r}$ are all positive, in consequence of (6.03). From the first of (6.02) we see that

$$
p_{r+1}<20 r p_{r} .
$$

Accordingly, we derive

$$
u_{r+1}<2 r u_{r} /(2 r+3),
$$

and thence

$$
\sum_{r=16}^{\infty} u_{r}<u_{15}\left(\frac{30}{33}+\frac{30}{33} \cdot \frac{32}{35}+\frac{30}{33} \cdot \frac{32}{35} \cdot \frac{34}{37}+\ldots\right)=30 u_{15},
$$

the last relation being a consequence of Gauss' formula for the hypergeometric function $F(15,1$; $\left.16 \frac{1}{2} ; 1\right)$; see [4], eq (15.1.20). Since

$$
30 u_{15}=0.0141823625 \text {. . }
$$

the stated result now follows.

\section{Clenshaw's Numerical Method for Ordinary Differential Equations}

As a final illustration, we consider a recurrence relation arising in the solution of a differential equation by the method of Clenshaw; compare Example 4 of [3], section 11. Suppose first that we are interested in the solution of the equation

$$
(2 r-1) f_{r-1}-12 r f_{r}+(2 r+1) f_{r+1}=0
$$

satisfying the conditions $f_{0}=1$ and $f_{r} \rightarrow 0$ as $r \rightarrow \infty$. Approximations to $f_{r}$ are provided by the function $f_{r}^{(7)}$ given to six decimal places in table 4 of [3] and to ten decimal places in table 5 of the same reference. Let us seek bounds for the magnitude of the truncation error

$$
\phi_{r}^{(7)}=f_{r}-f_{r}^{(7)} .
$$

As in the other examples, we have

$$
\phi_{r}^{(7)}=E_{7} p_{r}, \quad E_{7}=\sum_{s=7}^{\infty} \frac{e_{s}}{p_{s} p_{s+1}} .
$$

In the present case $p_{r}$ satisfies the difference eq (7.01), and $e_{r}=1 /(2 r+1)$. Numerical values of $p_{r}$ are given in table 4 of [3] for $r=0,1, \ldots, 8$. Applying Theorem 2 to the sequence $\left\{p_{r+7}\right\}$, we have

$$
\kappa=p_{8} / p_{7}=5.4389,
$$

correct to five significant figures, and

$$
a=\inf _{r \geqq 8} \frac{2 r-1}{2 r+1}=\frac{15}{17}, \quad b=\frac{15}{17}+\inf _{r \geqq 8} \frac{10 r+1}{2 r+1}=\frac{96}{17} .
$$

Accordingly,

$$
17 \lambda^{2}-96 \lambda+15=0, \quad \lambda=5.4862>\kappa .
$$

Hence

$$
p_{r} \geqq p_{7} \kappa^{r-7} \quad(r \geqq 7) .
$$

Substitution of the last inequality in the second of (7.03) yields

$$
\begin{aligned}
E_{7} & \leqq \frac{1}{p_{7}^{2}}\left(\frac{1}{15 \kappa}+\frac{1}{17 \kappa^{3}}+\frac{1}{19 \kappa^{5}}+\ldots\right) \\
& <\frac{1}{15 p_{7}^{2}\left(\kappa-\kappa^{-1}\right)}=0.67713 \times 10^{-10} .
\end{aligned}
$$

The corresponding bounds for the errors $\phi_{r}^{(7)}$ are given in the second column of table A below, in units of the tenth decimal place. They exceed only slightly the estimates for the errors obtained by subtracting the entries in the columns headed $f_{r}^{(12)}$ and $f_{r}^{(7)}$ in table 5 of [3], and given in the third column of table A.

TABLE A

\begin{tabular}{l|r|r|r|r|r}
\hline \hline$r$ & $0.67713 p_{r}$ & $\begin{array}{c}10^{10} \phi_{r}^{(7)} \\
\text { (estimated) }\end{array}$ & $0.11303 p_{r}$ & $-3735 f_{r}^{(7)}$ & $\begin{array}{c}10^{9} \epsilon_{r}^{(7)} \\
\text { (estimated) }\end{array}$ \\
\hline & 0 & 0 & 0 & -3735 & -3655 \\
1 & 1 & 1 & 0 & -322 & -315 \\
2 & 3 & 3 & 0 & -41 & -40 \\
3 & 13 & 13 & -6 & -6 & -3 \\
4 & 63 & 63 & 10 & -1 & 9 \\
5 & 325 & 324 & 54 & 0 & 54 \\
6 & 1723 & 1715 & 288 & 0 & 286 \\
7 & 9268 & 9228 & 1547 & 0 & 1540 \\
\hline
\end{tabular}

Secondly, suppose that we are interested in the solution of (7.01), $y_{r}$, say, which satisfies the condition

$$
\frac{1}{2} y_{0}+y_{1}+y_{2}+y_{3}+\ldots=1 \text {. }
$$

(This is the form in which the example was originally proposed in [3].) Then $y_{r}$ is related to $f_{r}$, defined above, by

$$
y_{r}=f_{r} / F, \quad F=\frac{1}{2} f_{0}+f_{1}+f_{2}+\ldots .
$$

We seek bounds for the errors

$$
\epsilon_{r}^{(7)}=y_{r}-y_{r}^{(7)} \quad(r=0,1, \ldots 7),
$$


$y_{r}^{(7)}=f_{r}^{(7)} / F_{7}, \quad F_{7}=\frac{1}{2} f_{0}^{(7)}+f_{1}^{(7)}+f_{2}^{(7)}+\ldots .+f_{6}^{(7)}$.

Using (7.02) and (7.08), we find that

$$
\epsilon_{r}^{(7)}=\epsilon_{r}^{(7,1)}-\epsilon_{r}^{(7,2)},
$$

where

$$
\epsilon_{r}^{(7,1)}=\frac{\phi_{r}^{(7)}}{F_{7}+\sigma_{7}}, \quad \epsilon_{r}^{(7,2)}=\frac{\sigma_{7} f_{r}^{(7)}}{F_{7}\left(F_{7}+\overline{\left.\sigma_{7}\right)}\right.},
$$

and

$$
\begin{aligned}
\sigma_{7}=\frac{1}{2} \phi_{0}^{(7)}+\phi_{1}^{(7)}+\phi_{2}^{(7)} & +\ldots+\phi_{7}^{(7)}+ \\
& +f_{8}+f_{9}+f_{10}+\ldots .
\end{aligned}
$$

In order to assess an upper bound for $\sigma_{7}$, we need an upper bound for

$$
f_{r}=p_{r} \sum_{s=r}^{\infty} \frac{1}{(2 s+1) p_{s} p_{s+1}}
$$

when $r \geqq 8$; compare [3], eq (5.03). To construct this, we make a second application of Theorem 2 to the sequence $\left\{p_{r+7}\right\}$. We have

$$
A=\sup _{r \geqq 8} \frac{2 r-1}{2 r+1}=1, \quad B=1+\sup _{r \geqq 8} \frac{10 r+1}{2 r+1}=6 .
$$

Accordingly

$$
\Lambda^{2}-6 \Lambda+1=0, \quad \Lambda=3+\sqrt{8}=5.8284>\kappa,
$$

where $\kappa$ is defined by (7.04). Hence

$$
p_{r} \leqq p_{7} \Lambda^{r-7} \quad(r \geqq 7) .
$$

Substituting this result and (7.05) in (7.14), we find that

$$
\begin{aligned}
f_{r} & \leqq \frac{\Lambda^{r-7}}{p_{7}} \sum_{s=r}^{\infty} \frac{1}{(2 \bar{s}+1) \kappa^{2 s-13}} \\
& <\frac{\Lambda^{r-7}}{p_{7}(2 r+1)\left(\kappa-\kappa^{-1}\right) \kappa^{2 r-14}}(r \geqq 7),
\end{aligned}
$$

and thence

$$
\sum_{r=8}^{\infty} f_{r}<\frac{\Lambda}{17 p_{7}\left(\kappa-\kappa^{-1}\right)\left(\kappa^{2}-\Lambda\right)}=2007 \times 10^{-10},
$$

correct to ten decimal places. Adding this value to $10^{-10}$ times the sum of the entries in the column of table A headed $0.67713 p_{r}$, we derive compare (7.13).

Since $F_{7}$ and $\sigma_{7}$ are both positive, we obtain from (7.03), (7.06), (7.12), and the value $F_{7}=0.59907 \mathrm{ex}$ tracted from [3], table 5

$$
\epsilon_{r}^{(7,1)} \leqq \frac{\phi_{r}^{(7)}}{F_{7}}=\frac{E_{7} p_{r}}{F_{7}} \leqq 0.11303 \times 10^{-9} p_{r}
$$

and

$$
\boldsymbol{\epsilon}_{r}^{(7,2)} \leqq \frac{\sigma_{7}}{F_{7}^{2}} f_{r}^{(7)} \leqq 3735 \times 10^{-9} f_{r}^{(7)},
$$

both $\epsilon_{r}^{(7,1)}$ and $\epsilon_{r}^{(7,2)}$ obviously being nonnegative. The values of $0.11303 p_{r}$ and $-3735 f_{r}^{(7)}$ are given in the fourth and fifth columns of table A. Comparison with the estimated values of $10^{9} \epsilon_{r}^{(7)}$, extracted from table 5 of [3] and given in the final column, is again fully satisfactory.

\section{Summary}

In the first part of this paper (secs. 1-4) simple lower and upper bounds are established for the solutions of second-order homogeneous linear difference equations, in ranges in which the solutions are exponential in character.

In the second part (secs. 5-7), the results are applied to an algorithm for the computation of subdominant solutions of second-order difference equations which was introduced recently by the writer. It is shown by means of examples how to compute strict and extremely realistic bounds for the truncation error associated with the algorithm. The bounds depend only on simple properties of the coefficients in the difference equation, and are independent of asymptotic theories of the solutions.

The writer is grateful to C. W. Clenshaw and G. F. Miller for valuable criticisms of an earlier form of this paper.

\section{References}

[1] Blanch, G., Numerical evaluation of continued fractions, Rev. Soc. Indust. Appl. Math. 6, 383-421 (1964).

[2] Merkes, E. P., On truncation errors for continued fraction computations, J. Numer. Anal. Soc. Indust. Appl. Math. 3, 486496 (1966).

[3] Olver, F. W. J., Numerical solution of second-order linear difference equations, J. Res. NBS 71B (Math. and Math. Phys.) Nos. 2 \& 3, 111-129 (1967).

[4] National Bureau of Standards, Handbook of mathematical functions, Appl. Math. Ser. 55 (Government Printing Office, Washington, D.C. 20402, 1964).

(Paper 71B4-238) 